\title{
NOVEL INSIGHTS INTO COMPLEX CARDIOVASCULAR PATHOLOGIES USING 4D FLOW ANALYSIS BY CARDIOVASCULAR MAGNETIC RESONANCE IMAGING
}

\section{SHORT TITLE: 4D FLOW BY CMR}

${ }^{1 *}$ Adam James Lewandowski DPhil, ${ }^{2}$ Betty Raman MBBS, ${ }^{3}$ Rajarshi Banerjee BMBCh, MPH, DPhil, ${ }^{3}$ Matteo Milanesi PhD

${ }^{1}$ Oxford Cardiovascular Clinical Research Facility and ${ }^{2}$ University of Oxford Centre for Clinical Magnetic Resonance Research, Division of Cardiovascular Medicine, Radcliffe Department of Medicine, University of Oxford, Oxford, United Kingdom ${ }^{3}$ Perspectum Diagnostics, Oxford, United Kingdom

*Corresponding author: Dr Adam James Lewandowski, Oxford Cardiovascular Clinical Research Facility, John Radcliffe Hospital, OX3 9DU. Oxford, United Kingdom.

Email: adam.lewandowski@cardiov.ox.ac.uk

Tel: $+44(0) 1865223680$ 


\begin{abstract}
Assessment of variations in blood flow is essential in order to fully understand cardiovascular function in disease pathologies and for identification of individuals at long-term risk of cardiovascular disease development. Qualitative and quantitative assessments of blood flow by imaging modalities have been limited, and much of the accurate quantification has relied on invasive measures. Four-dimensional velocity cardiovascular magnetic resonance (4D flow CMR) offers increasing potential for the non-invasive assessment of blood flow in the heart and major blood vessels such as the aorta. 4D flow CMR refers to phase contrast CMR with flow encoding in all three spatial directions that is resolved relative to all three dimensions of space and to the dimension of time throughout the cardiac cycle. It has been demonstrated that 4D flow CMR can be used to assess parameters such as flow, pressure, velocity, wall shear stress and turbulent kinetic energy throughout the heart and major vessels of the cardiovascular system. As such, it has been possible to gain new insights into cardiovascular pathologies such as, but not limited to, hypertrophic cardiomyopathy, dilated cardiomyopathy, Marfan syndrome and aortic bicuspid valve disease. Future work to standardize 4D flow CMR scan acquisition parameters is required, as is the development of automated analysis tools and standardized reporting of quantitative metrics to increase capacity for larger studies and translation to clinical practice. In doing so, the potential for 4D flow CMR to disentangle complex questions related to cardiovascular function will be maximized.
\end{abstract}




\section{INTRODUCTION}

Cardiovascular diseases remain among the leading causes of morbidity and mortality worldwide $(1,2)$. The burden to the patient's quality of life as a result of cardiovascular disease is enormous, as are the increasing financial pressures on global healthcare systems. Over several decades now, scientists and clinicians in the field of cardiovascular medicine have worked to advance novel strategies to identify individuals at greatest risk of developing cardiovascular disease and how best to manage patients in heart failure. Novel biomarkers have been established and applied to indicate changes in cardiovascular function, including imaging modalities. A significant emphasis has been placed on myocardial characterization and assessment of left ventricular function. However, the study of how changes in cardiovascular function impact blood flow, oxygen and nutrient delivery to the body, as well as the functional consequence of disrupted blood flow, has previously been limited. Indeed, echocardiography and cardiovascular magnetic resonance (CMR) imaging have predominantly focused on heart and blood vessel structural and functional changes.

Four-dimensional velocity CMR, or simply 4D flow CMR, has demonstrated increasing potential in both research and clinical scenarios. 4D flow CMR is three-directional, timeresolved flow encoded data in a three-dimensional volume $(3,4)$. More specifically, 4D flow CMR refers to phase contrast CMR with flow encoding in all three spatial directions that is resolved relative to the dimension of time along the cardiac cycle and to all three dimensions of space. As a result, the 4D flow CMR technique provides three-directional velocity encoded information (in addition to anatomical information) at each measured time point. In order to provide three-dimensional coverage, respiratory compensation approaches are implemented. This typically involves the use of respiration navigators or bellows, which allows the individual to breathe freely while obtaining images corresponding to specific phases of the 
respiratory cycle such that the final dataset appears to be collected during the same respiratory cycle $(4,5)$.

This technique has improved characterization of in vivo cardiovascular flow fields by combining visualization and quantification of flow parameters. For instance, in addition to being able to accurately determine aortic blood flow, velocity, and pressure in individuals with aortic bicuspid valve disease, it has been possible to visually identify altered helical flow directions in individuals with this inherited disease, which associate with more severe flow abnormalities and increased wall shear stress $(6,7)$.

This review briefly discusses the importance of gaining insight into heart and vessel blood flow to improve our understanding of adaptive and maladaptive changes in the cardiovascular system; applications of 4D flow CMR to research and clinical questions; as well as some of the key challenges, limitations and future work that may enable utilization of 4D flow CMR as a biomarker across the investigative spectrum of cardiovascular sciences.

\section{INSIGHT INTO BLOOD FLOW}

Pulsatile blood flow through the heart chambers and large vessels is multidimensional and multidirectional (8). It is increasingly understood that investigations into blood flow under both physiological and pathophysiological conditions reveal a wealth of information about cardiovascular disease progression. Under normal physiological conditions, the main purpose of the heart and major vessels is to lead the flowing blood throughout the body. The cardiovascular system is thus responsible for driving, regulating and upholding this process both at rest and under stress conditions. 
The influence of flow on cardiac remodeling is evident from the earliest stages of development: during fetal life - where a large proportion of flow bypasses the lungs and left ventricle via the foramen ovale and ductus arteriosus - flowing blood through the developing heart is largely responsible for the final cardiac geometry (9). These flow-induced processes continue to be vital throughout life, and in particular in the progression and pathophysiology of disease and cardiovascular remodeling. Transthoracic echocardiography and transesophageal echocardiography have long been the primary tools for diagnosis and monitoring of diseases such as congenital heart disease (10). However, image quality using echocardiography is determined by body habitus, presence of surgical scars and operator experience. Other methods to visualize the complex anatomy include either invasive approaches like catheter-based angiography or methods requiring the use of contrast as in the case of computed tomography (CT) angiography. 4D flow CMR permits the non-invasive assessment of complex cardiovascular anatomy and hemodynamics with the use of a larger field of view, offering greater anatomical coverage. Retrospective examination of specific regions of interest in three-dimensional volume datasets during specific phases of the cardiac cycle permits the identification of potential shunts or redirected blood flow with greater ease (11). It may also be possible to study the impact of cardiac intervention on cardiac flow (12). For instance, interrogation of 4D flow CMR datasets may be used to compare flow shunting in patients post Fontan repair $(13,14)$. Furthermore, flow abnormalities may provide early markers of poor prognosis in individuals to permit timely intervention to alter disease course.

Assessment of flow using imaging modalities has been limited, with the majority of measures being focused on morphological changes to describe cardiac function. Doppler echocardiography allows for assessment of blood flow velocities, though it is limited as a result of methodological constraints and assumptions applied to the calculations in situations 
of complex anatomical geometry or flow profiles (15). While Doppler echocardiography can be used to assess blood flow using the colour Doppler mode to provide a two-dimensional visualization of unidirectional flow velocity and can also be used to measure flow velocity in the direction of the ultrasound beam, limited acoustic windows and variable measurements due to operator expertise, patient body habitus and probe alignment are common shortcomings associated with this technique (15).

CMR-based two-dimensional flow methods have been used for over 30 years now. Twodimensional cine phase contrast CMR imaging uses velocity encoding in a single direction to calculate blood flow, but fails to capture the complex flow patterns that actually exist within the heart and blood vessels (16). As such, 4D flow CMR has been developed to capture this intricacy and has been applied to not only give insight into blood flow, velocity and pressure, but also to determine shear stress and energetics of flow.

\section{APPLICATIONS OF 4D FLOW}

4D flow CMR enables a wide variety of options for visualization and quantification of blood flow. This includes basic aspects of blood flow in the heart and blood vessels, such as flow volume and peak velocity, which are comparable to two-dimensional cine phase contrast CMR. More advanced features such as the estimation of hemodynamic effects at the vessel wall and myocardium, including wall shear stress, turbulent kinetic energy and pulse wave velocity, have also been described along with visualization techniques of flow pathways in the heart and great vessels (17). 


\section{Visualization of flow}

Visualization methods have been used to enhance our understanding of 4D flow CMR data. Streaklines, streamlines, and pathlines are all examples of flow visualization methods used to assess features of blood flow $(18,19)$. Streaklines refer to the location of particles at a given time that all passed through a particular point. Streamlines are lines representing the blood flow direction at any point in time, as these lines are tangent to the local velocity vectors at all spatial points along their length (18). As such, streamlines do not reflect the path traveled by individual blood cells in a pulsatile flow field and do not account for time-related flow variations. Pathlines, however, allow for the visualization of the path an imaginary, massless particle through space and time would take $(19,20)$. The difficulty arises in determining the best visualization modality for the question at hand, though, in most cases, multiple approaches to enhance the qualitative understanding of the quantified 4D flow CMR data are beneficial. Calkoen et al showed the utility of streamline visualization by demonstrating that it improved quantification of trans-left atrioventricular valve blood flow quantification by optimizing the measurement plane (21). In doing so, they were able to quantify the change in dynamic trans-left atrioventricular valve blood inflow and increased lateral flow after septal defect repair.

\section{Quantifying intra-cardiac flow}

4D flow CMR analysis is commonly applied to the left ventricle to quantify intra-cardiac flow (22). 4D flow within the left ventricle has been quantified and separated into four different functional flow components to better understand ventricular function: direct blood flow, retained inflow, delayed ejection flow, and residual volume (Figure 1) (23). Direct flow refers to the blood that enters the heart during diastole from the left atrium and is then ejected through to the aorta via the aortic valve during systolic left ventricular contraction within the 
same cardiac cycle. Retained inflow refers to the blood that enters the heart during diastole from the left atrium, but then does not leave the left ventricle in the same cardiac cycle. It is therefore only a component of the inflow volume. Delayed ejection flow refers to blood flow that is already in the left ventricle at the start of diastole of the cardiac cycle under question, but then gets ejected through the aortic valve to the aorta during systole of the same cardiac cycle. It is therefore only a component of the outflow volume. Residual volume refers to blood flow that resides within the left ventricle at the start of diastole and does not get ejected during systole of the same cardiac cycle. It therefore refers to blood that resides within the left ventricle for at least two cardiac cycles and is neither a component of inflow or outflow volume.

Kinetic energy can be calculated for each of the aforementioned functional flow components to give unique insight into subclinical left ventricular dysfunction. For instance, Eriksson et al. demonstrated that, despite equivalent stroke volumes, the blood flow paths and energetics between healthy individuals and those with dilated cardiomyopathy differed significantly (22). This component examination approach to intra-cardiac blood flow analysis using 4D flow CMR therefore allows for more detailed insight into the underlying function and adaptations in the remodeled heart.

\section{Four-dimensional phase-contrast magnetic resonance angiography}

Vascular geometry may also be estimated from four-dimensional phase contrast magnetic resonance angiography (4D PC MRA), where the outline of the surface imaged can be obtained for visualization and volumetric analysis. Although limited by lower spatial resolution, 4D PC MRA may provide an approximation of the vascular geometry without the 
need for contrast agents (24). This is particularly beneficial for individuals with contraindications to contrast administration or those requiring repeated scans.

\section{Intra-cardiac pressure}

4D flow CMR also offers the significant advantage of being able to calculate intra-cardiac pressure. While the gold standard continues to be invasive measures using pressure transducers to calculate absolute pressure, non-invasive modalities have been limited in their use for calculating pressure differences and relative pressure. By combining the 4D flow CMR data with the anatomical left ventricular data, relative pressure fields can be calculated. Segmentation of the left ventricle at end diastole allows for determination of the boundaries to be used in combination with the 4D flow data to calculate the relative pressure fields (25). 4D flow CMR was demonstrated by van Ooij et al to be able to detect altered pressure gradients and energy loss in individuals with hypertrophic cardiomyopathy compared to controls, indicating significantly greater left ventricular loading (26).

\section{Turbulent kinetic energy}

Calculation of turbulent kinetic energy is of interest because it results in loss of energy and exposure of the surrounding tissue (i.e. the ventricular wall) to abnormal shear stress and mechanical forces. 4D flow CMR allows for the quantification of turbulent kinetic energy, which is dissipated as heat and therefore seen as a measure of inefficiency. By calculating turbulent kinetic energy, Zajac et al were able to demonstrate that turbulent kinetic energy loss in the normal, healthy heart ranges from 0 to $5 \mathrm{~mJ}$ (27). Furthermore, they found that in individuals with dilated cardiomyopathy, early diastolic (E wave) turbulent kinetic energy did not differ from healthy controls, though late diastolic (A wave) turbulent kinetic energy values were much greater in individuals with dilated cardiomyopathy. 


\section{Helical aortic flow and wall shear stress}

Perhaps the most extensively studied application of 4D flow CMR is in the assessment of aortic flow. Quantification and visualization of flow in three dimensions in the aorta enables the complex assessment of fluid dynamics. As a result, novel hemodynamic parameters that may impact on cardiac and vascular remodeling such as helical flow assessment, wall sheer stress and flow jet eccentricity can now be assessed from 4D flow CMR datasets (28-31).

Helical aortic flow has both a forward component along the long axis of the aorta and a rotational component along the long axis of the aorta in a circumferential direction (6). In healthy young individuals, flow through the ascending aorta is unified with only a slight helical component. However, ageing and pathologies that affect the anatomy or function of the aorta are likely to disrupt laminar flow. As such, helical flow can be measured using the circumferential circulation measure, which is calculated as the integral of vorticity with respect to the cross-sectional area through the aorta (32). Helical flow is normally visualized using streamlines and particle traces, and defined as either left or right handed (28). Improvements in spatial resolution and acquisition time have also enabled the estimation of sheer stress from the viscosity of fluid, three-dimensional velocity and unit inward surface normal vector. The clinical utility of this measure has recently been demonstrated in individuals with dilated ascending aorta where wall sheer stress was found to directly correlate with changes in blood flow and velocity (33). In order to calculate wall shear stress in the aorta, multiple axial slices are taken along the region of interest (i.e. ascending aorta), with user-defined points on each slice along the boundary of the aortic wall (33). A centroid point is then automatically determined, and from this and the points along the boundary of the vessel wall, multiple surface points are determined. From each surface point, a unit inward normal vector and velocity vector field can be calculated. The cross product of each 
velocity vector and the unit inward surface normal vector are then taken in order to obtain the velocity component tangent to the vessel surface. Wall shear stress is then calculated as a combination of the viscosity of fluid, three-dimensional velocity and unit inward surface normal vector. Bieging et al have improved this technique by increasing spatial resolution and decreasing image acquisition time (33). They demonstrated that, in individuals with dilated ascending aortas, the change in flow and velocity in the ascending aorta was proportional to wall shear stress. Wall shear stress was increased in diastole, with a delayed onset in systole and reduced pulsatility throughout the cardiac cycle.

An area of growing research interest and debate pertains to the influence of fluid dynamics on aortic remodeling and aneurysm formation in aortic bicuspid valve disease (34). There is sufficient data suggesting that altered valve geometry in aortic bicuspid valve disease is associated with high velocity flow jets, which impinge on the wall and result in increased wall sheer stress in the region $(6,28,32)$. It has also been demonstrated that there is an increase in helical flow in individuals with aortic bicuspid valve disease (28), and that particular helical flow directions associate with more severe flow abnormalities and increased wall shear stress (6). The observation that distinct flow characteristics exist amongst individuals with aortic bicuspid valves may enable risk stratification of individuals into those that are likely to develop a progressive aortopathy and require surgical intervention in the future. Use of $4 \mathrm{D}$ flow CMR to assess helical aortic flow may therefore prove to be a clinically useful tool for prognostication of patients with aortic bicuspid valve disease.

\section{CHALLENGES, LIMITATIONS AND FUTURE WORK}

4D flow CMR has developed rapidly over the past few years, gaining momentum in the research field. This has resulted in individual sites developing their own sequences and 
analysis tools, making it difficult to combine and compare data across centres and within particular disease states. Standardization of protocols will be essential for moving this imaging tool to clinical practice and wider clinical use given the many steps involved in producing high quality data (Figure 2). Furthermore, the amount of data collected in each 4D flow CMR dataset is substantial. The data acquisition sequence is long (generally 10-15 minutes), resulting in data capture over several thousand cardiac cycles. While ways to reduce acquisition sequence time are continuously being explored, data analysis remains technically challenging because of the wealth of information available. As such, ways to automate analysis would be helpful (35), though directed investigations to the research or clinical questions at hand will continue to be required to manage the analysis process. Commercially available standardized acquisition and analysis of 4D flow are available on some MR platforms (i.e. Arterys, USA (GE platform)), and offer the promise of rapid and consistent analysis of large datasets with quality assurance; a key requirement for translating the research potential into clinical utility.

Balancing improvements in acquisition time with higher spatial resolution and higher temporal resolution poses several challenges. Low spatial resolution makes it difficult to calculate parameters such as wall shear stress, while low temporal resolution may result in missing perturbations in flow throughout the cardiac cycle that provide insight into cardiac function (36). However, increasing spatial and temporal resolution comes at the cost of substantially lengthening scan sequence acquisition times (37). To overcome this, techniques to shorten scan acquisition times have been used $(36,38,39)$, though this ultimately results in loss of signal to noise. Hess et al have demonstrated that this can be overcome by scanning at higher field strengths (37). They showed that scanning at 7 Tesla field strengths resulted in a 2.2-fold increase in signal to noise compared to scanning at 3 Tesla, and a 1.7 fold increase in 
signal to noise compared to scanning at 1.5 Tesla. Further improvements in signal to noise were also observed when using contrast enhancement at each of the different field strengths. Other measures to reduce scan times include the use of high performance gradients, which in turn reduce echo and repetition times. The use of parallel imaging, phased array coils and multi-receiver channels, as well as advanced accelerated imaging such as radial under sampling, kt-BLAST (broad-use linear acquisition speed-up technique), kt-SENSE (sensitivity encoding), kt-GRAPPA (generalized autocalibrating partially parallel acquisitions) or compressed sensing also help reduce scan times (40-46).

4D flow CMR is commonly plagued by various sources of error, such as phase offset errors, which are frequent and can disrupt image quality and reduce accuracy of measurements (47). Other sources of error are also common due to Eddy currents, Maxwell terms and gradient field non-linearity errors. Appropriate corrections must be applied before the procession of 4D flow CMR data sets. For example, Maxwell and gradient non-linearity corrections can be applied during reconstruction where as Eddy current corrections need to be integrated during data analysis (48).

Inaccuracies in flow estimation and quantification may also result from the phenomenon of velocity aliasing. This is likely to occur if the maximum velocity or velocity encoding (VENC) that is set is lower than the true physiological velocity, resulting in an underestimation of flow components. To avoid this, velocity should be set by the operator to optimally capture both physiological and pathophysiological alterations in blood flow in three-dimensional volumes. As increasing velocity comes at a cost of increasing noise, cautious selection of approximate velocities is therefore often required (49). 
Examples of VENC for physiological conditions include $50-80 \mathrm{~cm} / \mathrm{s}$ in large vessels, $150-$ $200 \mathrm{~cm} / \mathrm{s}$ in the aorta and $100-15 \mathrm{~cm} / \mathrm{s}$ for intra-cardiac flow. For pathophysiological conditions such as aortic stenosis or hypertrophic cardiomyopathy, velocities of $250-400$ $\mathrm{cm} / \mathrm{sec}$ may be needed. Further consideration should also be given to the blood volume and specific clinical questions.

The possibilities for use of $4 \mathrm{D}$ flow CMR in the future to disentangle complex disease pathologies and identify individuals at future cardiovascular risk are vast. Combining this data with more comprehensive modalities of capturing three-dimensional geometry from CMR, such as cardiac statistical atlas formation (50,51), provides potential for further determining the interaction between cardiac remodeling and changes in 4D flow CMR parameters. This also offers potential for longitudinal studies to assess this interaction and change with disease progression or in response to therapeutic interventions, such as pharmacological treatment. Long-term clinical outcome studies will be required to identify the additional risk associated with particular 4D flow CMR changes and ability of these changes to predict clinical events. In valvular disease in particular, this should be possible in the near future with well-characterized cohorts of patients with, for example, mitral regurgitation or bicuspid aortic valve disease. With multi-organ diseases, such as hypertension, the impact of flow assessment in the aorta and great vessels may help to stratify high-risk patients and those most likely to benefit from preventive therapies, such as elective aneurysm repair.

\section{CONCLUSION}

4D flow CMR is an exciting new area of cardiovascular imaging. While it has been around for over two decades, the field has gained substantial momentum in recent years. 4D flow 
CMR offers significant potential for quantification and visualization of blood flow in the heart and major blood vessels of the human body. New insights into disease pathologies have already resulted from use of this technique, and will continue to come together with advances in sequence resolution, usability and application to novel areas of cardiovascular sciences.

\section{CONFLICTS OF INTEREST}

There are no conflicts of interest to disclose.

\section{ACKNOWLEDGEMENTS}

Dr AJ Lewandowski is funded by a British Heart Foundation Project Grant (PG/13/58/30397). 


\section{REFERENCES}

1. Pagidipati NJ, Gaziano TA. Estimating Deaths From Cardiovascular Disease: A Review of Global Methodologies of Mortality Measurement. Circulation 2013;127:749.

2. Nichols M, Townsend N, Scarborough P, Rayner M. Cardiovascular disease in Europe 2014: epidemiological update. European Heart Journal 2014;35:2950.

3. Stankovic Z, Allen BD, Garcia J, Jarvis KB, Markl M. 4D flow imaging with MRI. Cardiovascular Diagnosis and Therapy 2014;4:173-192.

4. Markl M, Chan FP, Alley MT et al. Time-resolved three-dimensional phase-contrast MRI. Journal of Magnetic Resonance Imaging 2003;17:499-506.

5. Mark1 M, Harloff A, Bley TA et al. Time-resolved 3D MR velocity mapping at 3T: Improved navigator-gated assessment of vascular anatomy and blood flow. Journal of Magnetic Resonance Imaging 2007;25:824-831.

6. Bissell MM, Hess AT, Biasiolli L et al. Aortic Dilation in Bicuspid Aortic Valve Disease. Circulation: Cardiovascular Imaging 2013;6:499.

7. Lamata P, Pitcher A, Krittian S et al. Aortic relative pressure components derived from four-dimensional flow cardiovascular magnetic resonance. Magnetic Resonance in Medicine 2014;72:1162-1169.

8. Richter Y, Edelman ER. Cardiology Is Flow. Circulation 2006;113:2679.

9. Taber LA. Mechanical aspects of cardiac development. Progress in Biophysics and Molecular Biology 1998;69:237-255.

10. Warnes CA, Williams RG, Bashore TM et al. ACC/AHA 2008 Guidelines for the Management of Adults With Congenital Heart Disease: Executive Summary. Circulation 2008;118:2395.

11. François CJ, Srinivasan S, Schiebler ML et al. 4D cardiovascular magnetic resonance velocity mapping of alterations of right heart flow patterns and main pulmonary artery hemodynamics in tetralogy of Fallot. Journal of Cardiovascular Magnetic Resonance 2012;14:16.

12. Geiger J, Markl M, Jung B et al. 4D-MR flow analysis in patients after repair for tetralogy of Fallot. European Radiology 2011;21:1651-1657.

13. Markl M, Geiger J, Kilner PJ et al. Time-resolved three-dimensional magnetic resonance velocity mapping of cardiovascular flow paths in volunteers and patients with Fontan circulation. European Journal of Cardio-Thoracic Surgery 2011;39:206212.

14. Bächler P, Valverde I, Pinochet N et al. Caval Blood Flow Distribution in Patients with Fontan Circulation: Quantification by Using Particle Traces from 4D Flow MR Imaging. Radiology 2013;267:67-75.

15. Fyrenius A, Wigström L, Bolger AF et al. Pitfalls in Doppler Evaluation of Diastolic Function: Insights from 3-Dimensional Magnetic Resonance Imaging. Journal of the American Society of Echocardiography;12:817-826.

16. Underwood SR, Firmin DN, Klipstein RH, Rees RS, Longmore DB. Magnetic resonance velocity mapping: clinical application of a new technique. British Heart Journal 1987;57:404-412.

17. Dyverfeldt P, Bissell M, Barker AJ et al. 4D flow cardiovascular magnetic resonance consensus statement. Journal of Cardiovascular Magnetic Resonance 2015;17:72.

18. Napel S, Lee DH, Frayne R, Rutt BK. Visualizing three-dimensional flow with simulated streamlines and three-dimensional phase-contrast MR imaging. Journal of Magnetic Resonance Imaging 1992;2:143-153. 
19. Buonocore MH. Visualizing blood flow patterns using streamlines, arrows, and particle paths. Magnetic Resonance in Medicine 1998;40:210-226.

20. Wigström L, Ebbers T, Fyrenius A et al. Particle trace visualization of intracardiac flow using time-resolved 3D phase contrast MRI. Magnetic Resonance in Medicine 1999;41:793-799.

21. Calkoen EE, Roest AAW, Kroft LJM et al. Characterization and improved quantification of left ventricular inflow using streamline visualization with 4DFlow MRI in healthy controls and patients after atrioventricular septal defect correction. Journal of Magnetic Resonance Imaging 2015;41:1512-1520.

22. Eriksson J, Bolger AF, Ebbers T, Carlhäll C-J. Four-dimensional blood flow-specific markers of LV dysfunction in dilated cardiomyopathy. European Heart Journal Cardiovascular Imaging 2013;14:417.

23. Bolger AF, Heiberg E, Karlsson M et al. Transit of blood flow through the human left ventricle mapped by cardiovascular magnetic resonance. Journal of Cardiovascular Magnetic Resonance 2007;9:741-747.

24. Frydrychowicz A, François CJ, Turski PA. Four-dimensional phase contrast magnetic resonance angiography: Potential clinical applications. European Journal of Radiology 2011;80:24-35.

25. Eriksson J, Bolger AF, Carlhäll C-J, Ebbers T. Spatial heterogeneity of fourdimensional relative pressure fields in the human left ventricle. Magnetic Resonance in Medicine 2015;74:1716-1725.

26. van Ooij P, Allen BD, Contaldi C et al. 4D flow MRI and T1-Mapping: Assessment of altered cardiac hemodynamics and extracellular volume fraction in hypertrophic cardiomyopathy. Journal of Magnetic Resonance Imaging 2016;43:107-114.

27. Zajac J, Eriksson J, Dyverfeldt P, Bolger AF, Ebbers T, Carlhäll C-J. Turbulent kinetic energy in normal and myopathic left ventricles. Journal of Magnetic Resonance Imaging 2015;41:1021-1029.

28. Hope MD, Hope TA, Meadows AK et al. Bicuspid Aortic Valve: Four-dimensional MR Evaluation of Ascending Aortic Systolic Flow Patterns. Radiology 2010;255:5361.

29. Bürk J, Blanke P, Stankovic Z et al. Evaluation of 3D blood flow patterns and wall shear stress in the normal and dilated thoracic aorta using flow-sensitive 4D CMR. Journal of Cardiovascular Magnetic Resonance 2012;14:84-84.

30. Geiger J, Arnold R, Herzer L et al. Aortic wall shear stress in Marfan syndrome. Magnetic Resonance in Medicine 2013;70:1137-1144.

31. Hope MD, Hope TA, Crook SES et al. 4D Flow CMR in Assessment of ValveRelated Ascending Aortic Disease. JACC: Cardiovascular Imaging 2011;4:781-787.

32. Hess AT, Bissell MM, Glaze SJ et al. Evaluation of Circulation, $\Gamma$, as a quantifying metric in 4D flow MRI. Journal of Cardiovascular Magnetic Resonance 2013;15:E36.

33. Bieging ET, Frydrychowicz A, Wentland A et al. In vivo three-dimensional MR wall shear stress estimation in ascending aortic dilatation. Journal of Magnetic Resonance Imaging 2011;33:589-597.

34. Barker AJ, Markl M, Bürk J et al. Bicuspid Aortic Valve Is Associated With Altered Wall Shear Stress in the Ascending Aorta. Circulation: Cardiovascular Imaging 2012;5:457.

35. Bustamante M, Petersson S, Eriksson J et al. Atlas-based analysis of 4D flow CMR: Automated vessel segmentation and flow quantification. Journal of Cardiovascular Magnetic Resonance 2015; 17:87. 
36. Stalder AF, Dong Z, Yang Q et al. Four-dimensional flow-sensitive MRI of the thoracic aorta: 12- versus 32-channel coil arrays. Journal of Magnetic Resonance Imaging 2012;35:190-195.

37. Hess AT, Bissell MM, Ntusi NAB et al. Aortic 4D flow: Quantification of signal-tonoise ratio as a function of field strength and contrast enhancement for $1.5 \mathrm{~T}, 3 \mathrm{~T}$, and 7T. Magnetic Resonance in Medicine 2015;73:1864-1871.

38. Jhooti P, Keegan J, Firmin DN. A fully automatic and highly efficient navigator gating technique for high-resolution free-breathing acquisitions: Continuously adaptive windowing strategy. Magnetic Resonance in Medicine 2010;64:1015-1026.

39. Kwak Y, Nam S, Akçakaya M et al. Accelerated aortic flow assessment with compressed sensing with and without use of the sparsity of the complex difference image. Magnetic Resonance in Medicine 2013;70:851-858.

40. Gu T, Korosec FR, Block WF et al. PC VIPR: A High-Speed 3D Phase-Contrast Method for Flow Quantification and High-Resolution Angiography. American Journal of Neuroradiology 2005;26:743-749.

41. Johnson KM, Lum DP, Turski PA, Block WF, Mistretta CA, Wieben O. Improved 3D phase contrast MRI with off-resonance corrected dual echo VIPR. Magnetic Resonance in Medicine 2008;60:1329-1336.

42. Tariq U, Hsiao A, Alley M, Zhang T, Lustig M, Vasanawala SS. Venous and Arterial Flow Quantification, are Equally Accurate and Precise with Parallel Imaging Compressed Sensing 4D Phase Contrast MRI. Journal of magnetic resonance imaging : JMRI 2013;37:1419-1426.

43. Baltes C, Kozerke S, Hansen MS, Pruessmann KP, Tsao J, Boesiger P. Accelerating cine phase-contrast flow measurements using k-t BLAST and k-t SENSE. Magnetic Resonance in Medicine 2005;54:1430-1438.

44. Moftakhar R, Aagaard-Kienitz B, Johnson K et al. Noninvasive Measurement of Intra-Aneurysmal Pressure and Flow Pattern Using Phase Contrast with Vastly Undersampled Isotropic Projection Imaging. American Journal of Neuroradiology 2007;28:1710-1714.

45. Stadlbauer A, van der Riet W, Crelier G, Salomonowitz E. Accelerated time-resolved three-dimensional MR velocity mapping of blood flow patterns in the aorta using SENSE and k-t BLAST. European Journal of Radiology 2010;75:e15-e21.

46. Lustig M, Donoho D, Pauly JM. Sparse MRI: The application of compressed sensing for rapid MR imaging. Magnetic Resonance in Medicine 2007;58:1182-1195.

47. Markl M, Bammer R, Alley MT et al. Generalized reconstruction of phase contrast MRI: Analysis and correction of the effect of gradient field distortions. Magnetic Resonance in Medicine 2003;50:791-801.

48. Walker PG, Cranney GB, Scheidegger MB, Waseleski G, Pohost GM, Yoganathan AP. Semiautomated method for noise reduction and background phase error correction in MR phase velocity data. Journal of Magnetic Resonance Imaging 1993;3:521-530.

49. Pelc NJ, Herfkens RJ, Shimakawa A, Enzmann DR. Phase contrast cine magnetic resonance imaging. Magnetic Resonance Quarterly 1991;7:229-254.

50. Lewandowski AJ, Augustine D, Lamata P et al. Preterm Heart in Adult Life: Cardiovascular Magnetic Resonance Reveals Distinct Differences in Left Ventricular Mass, Geometry, and Function. Circulation 2013;127:197.

51. Lamata P, Sinclair M, Kerfoot E et al. An automatic service for the personalization of ventricular cardiac meshes. Journal of The Royal Society Interface 2013;11. 


\section{FIGURES}

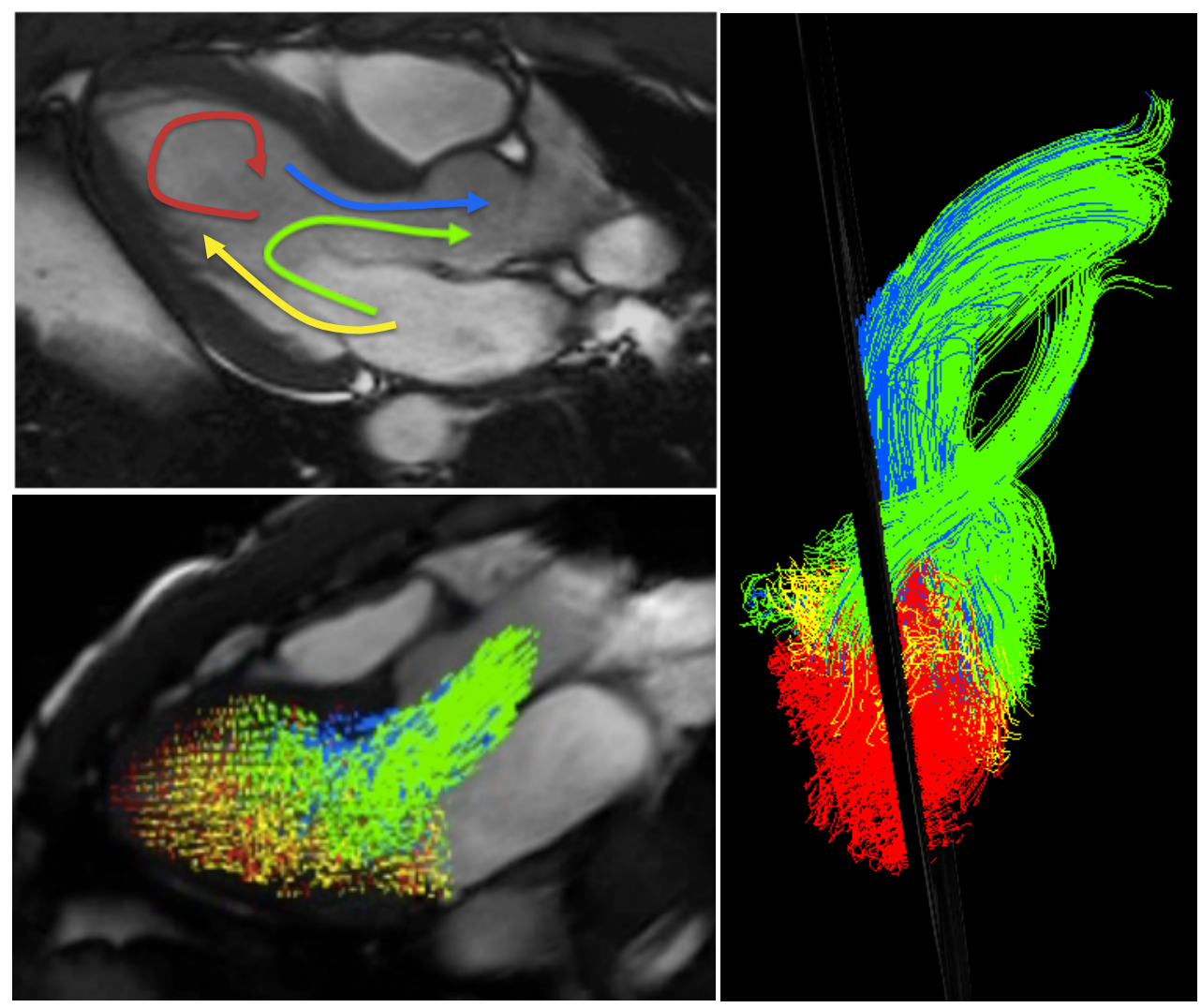

Figure 1 - Components of left ventricular blood flow. The top left panel shows a threechamber view of the left ventricle with coloured arrows representing the four flow components that make up the end-diastolic volume. The four components are direct blood flow (green), retained inflow (yellow), delayed ejection flow (red), and residual volume (blue). The bottom left panel shows a three-chamber view of the left ventricle with pathline visualization of the four flow components. The right panel shows a three-dimensional visualization of the pathlines emitted from the right ventricle relative to the left ventricle. 


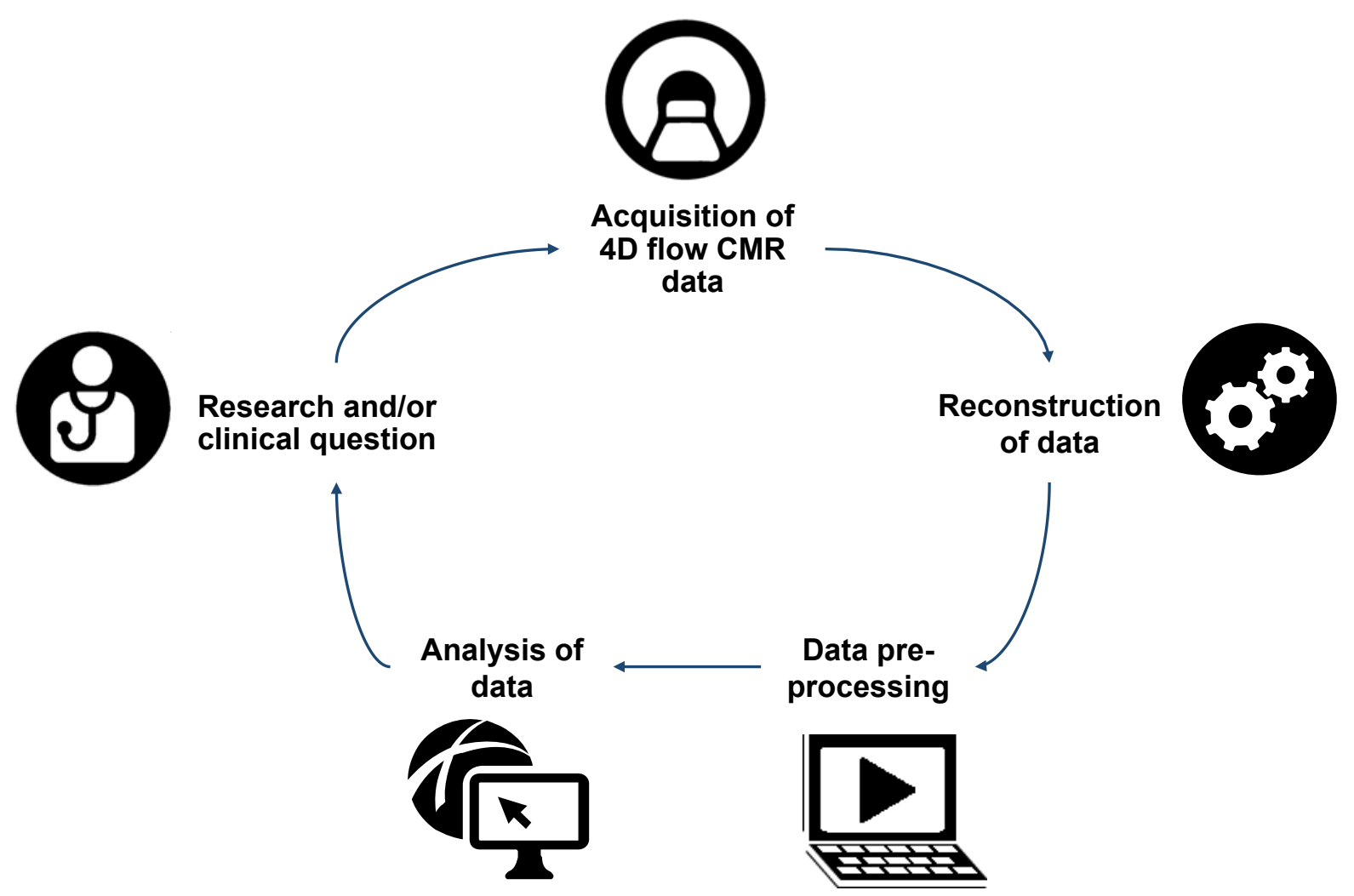

Figure 2 - Typical workflow for 4D flow CMR data acquisition and analysis for research and clinical applications. 